Theorem 1 is measured by the identification space $M^{*}$ whose elements are the leaves of $N$ and the components of $M-N$. If $M^{*}$ is metrizable, it can be shown to have inductive dimension 1 . In this case the argument above can be replaced by an application of the Vietoris mapping theorem.

\title{
BIBLIOGRAPHY
}

1. W. Ambrose and I. M. Singer, A theorem on holonomy, Trans. Amer. Math. Soc. 75 (1953), 428-443.

2. E. Cartan, Leçons sur la géométrie des espaces de Riemann, Gauthier-Villars, Paris, 1951.

3. S. Chern and R. Lashof, On the total curvature of immersed manifolds, Amer. J. Math. 79 (1957), 306-318.

4. P. Hartman and L. Nirenberg, On spherical image maps whose Jacobians do not change sign, Amer. J. Math. 81 (1959), 901-920.

University of California, Los Angeles

\section{ON THE EMBEDDABILITY OF THE REAL PROJECTIVE SPACES ${ }^{1}$}

\section{MARK MAHOWALD ${ }^{2}$}

In a paper of the same title, Massey [4] proved that if $2^{k-1}+2^{k-9}$ $-1 \leqq n<2^{k}$ then $P_{n}$ cannot be differentiably embedded in $R^{2^{k}}$. By using the technique of Massey in a different way we can prove the following theorem which clearly includes Massey's.

TheOREM. If $2^{k-1}<n<2^{k}$ then $P_{n}$ cannot be embedded differentiably in Euclidean space of dimension $2^{k}$.

Besides the result of Massey, the main result in this direction is if $2^{k-1}<n<2^{k}$ then $P_{n}$ cannot be embedded differentiably in $R^{2^{k}-1}$. Our result yields, in particular, that for $P_{2^{k}+1}$, the embedding in $R^{2^{k+1}+1}$ given by Hopf and James [1] is the best possible.

The following information from $[3 ; 4]$ will be needed. Let $M$ be a $n$-manifold differentiably embedded in $R^{n+k+1}$; and let $p: E \rightarrow M$ denote the bundle of unit normal vectors. Then there exist subalgebras $A^{*}(E, Z) \subset H^{*}(E, Z)$ and $A^{*}\left(E, Z_{2}\right) \subset H^{*}\left(E, Z_{2}\right)$ which satisfy the following conditions:

1. $A^{0}(E, G)=H^{0}(E, G)$,

2. $H^{q}(E, G)=A^{q}(E, G)+p^{*}\left(H^{q}(B, G)\right)(0<q<n+k)$,

3. $A^{q}(E, G)=0, q \geqq n+k$,

Received by the editors October 2, 1961.

${ }^{1}$ Sponsored by the U. S. Army Research Office (Durham).

2 The referee has informed me that the result of this paper has been obtained independently by Mr. J. P. Levine in his thesis at Princeton University. 
where $G=Z$ or $Z_{2}$. Moreover the algebra $A^{*}$ is closed under all natural cohomology operations.

By the well-known theorem of Seifert and Whitney, the characteristic class of a normal bundle vanishes hence the Gysin sequence breaks up into parts of length three, $0 \rightarrow H^{i+k}(M) \rightarrow \rightarrow^{*} H^{i+k}(E)$ $\rightarrow \psi H^{i}(M) \rightarrow 0$. Because of (2) $\psi$ must be an isomorphism on $A^{*}$. Each element in $H^{*}(E)$ can be written in the form $p^{*}\left(b_{1}\right)+a \cdot p^{*}\left(b_{2}\right)$ where $a$ is the unique element in $A^{k}$ such that $\psi(a)=1$. For simplicity we will suppress the map $p^{*}$ and write a general element in $H^{*}(E)$ as $b_{1}+a \cdot b_{2}$.

The proof of the theorem will consist in showing that $P_{m}\left(m=2^{k-1}+1\right)$ cannot be differentiably embedded in $R^{2^{k}}$. The result will then follow from the fact that $P_{m}$ can be differentiably embedded in $P_{n}$ for $n>2^{k-1}$.

A simple computation shows that the Stiefel-Whitney classes for the tangent bundle for $P_{2^{k-1}+1}$ are as follows: $W_{0}=1, W_{2}=\alpha^{2}, W_{m-1}$ $=\alpha^{m-1}$ where $\alpha$ is the nonzero element of $H^{1}\left(P_{m}, Z_{2}\right)$ and all other $W_{i}=0$. Using the fact that $W \cdot \bar{W}=1$ we see that $\bar{W}_{2 i}=\alpha^{2 i}, 0 \leqq i$ $\leqq(m-3) / 2$ and $\bar{W}_{j}=0$ for all other $j$.

Suppose $P_{m}$ is differentiably embedded in $R^{2^{k}}$. Let $E$ be the bundle of unit normal vectors over $P_{m}$ for this embedding. Then $E$ is a $(m-3)$-sphere bundle and since $\bar{W}_{m-2}=0$, the characteristic class vanishes. Let $a \in A^{m-3}$ be the element such that $\psi(a)=1$. Suppose that the nonzero element of $A^{m-2}$ is of the form $\alpha^{m-2}+a \cdot \alpha$; then, since $S q^{1}\left(\alpha^{m-2}+a \cdot \alpha\right)=\alpha^{m-1}+a \cdot \alpha^{2}$ because $a$ is an integer class, we have that the nonzero element of $A^{m-1}$ must be $\alpha^{m-1}+a \cdot \alpha^{2}$. But $\left(\alpha^{m-2}+a \cdot \alpha\right)\left(\alpha^{m-1}+a \cdot \alpha^{2}\right)=a \cdot \alpha^{m}+a^{2} \cdot \alpha^{3}+a \cdot \alpha^{m}$. This element must be in $A^{2 m+3}$ which is zero by (3).

But it equals $a^{2} \cdot \alpha^{3}=\left(S q^{m-3} a\right) \cdot \alpha^{3}=a \cdot \bar{W}_{m-3} \cdot \alpha^{3}$ by a result due to Liao [2], and this equals $a \cdot \alpha^{m} \neq 0$. Hence the nonzero element of $A^{m-2}$ is of the form $a \cdot \alpha$. But then $S q^{1} a \cdot \alpha=a \cdot \alpha^{2}$ is the nonzero element of $A^{m-1}$ and $\left(a \cdot \alpha^{2}\right)\left(a \cdot \alpha^{1}\right) \in A^{2 m-3}=0$ but it, as before, equals $a \cdot \alpha^{m}$ which is not zero.

\section{REFERENCES}

1. I. M. James, Some embeddings of projective spaces, Proc. Cambridge Philos. Soc. 55 (1959), 294-298.

2. S. D. Liao, On the theory of obstructions of fiber bundles, Ann. of Math. (2) 60 (1954), 146-191.

3. W. S. Massey, On the cohomology ring of a sphere bundle, J. Math. Mech. 7 (1958), 265-290.

4. - On the embeddability of the real projective spaces, Pacific J. Math. 9 (1959), 783-789.

SyRACUSE UNIVERSITY 